\title{
Over Half a Century of Research in Oxides
}

\author{
K. A. Müller
}

Received: 10 January 2015 / Accepted: 12 January 2015 / Published online: 31 January 2015

(c) Springer Science+Business Media New York 2015

An attempt will be made to recall some of the highlights of the research in oxides achieved by the author and various collaborators over a period of 58 years. These were: the observation of the paramagnetic resonance of transition metal ions of high valency like $\mathrm{Fe}^{3+}, \mathrm{Fe}^{4+}, \mathrm{Fe}^{5+}$ and $\mathrm{Co}^{4+}$ and their photochromic behaviour; the detection of $\mathrm{Mn}^{4+}$ in $\mathrm{BaTiO}_{3}$ and the proof for the presence of order-disorder character in these ferroelectric transitions, and then the temperature dependence of the rotational order parameter of the structural phase transitions in $\mathrm{LaAlO}_{3}$ and $\mathrm{SrTiO}_{3}$. Near the transition, the observation of critical behaviour compatible with renormalisation group theory and the presence of a Potts transition line, a critical end point and a Lifshitz point under uniaxial stress in $\mathrm{SrTiO}_{3}$ and $\mathrm{CaGdF}_{3}$. This will be followed by the determination of the stabilisation energy ofthe Jahn-Teller effect with octahedral oxygen neighbours to various ions; leading over to the concept of Jahn-Teller polarons as quasi particles for the generation of superconductivity, and the experimental observation of Jahn-Teller bipolarons and their stabilisation energy determined in one of the HTS copper oxides.

A comprehensive collection of relevant papers appeared in the book "Properties of Perovskites and Other Oxides" by K.A. Müller and T.W. Kool (World Scientific, 2010).

K. A. Müller ( $ه)$

Physik Institut, University of Zürich, Zürich, Switzerland

e-mail: irmamf@bluewin.ch 